\title{
Multiple Oscillatory Modes of the Argentine Basin. Part I: Statistical Analysis
}

\author{
WILBERT WEIJER \\ Scripps Institution of Oceanography, La Jolla, California, and Los Alamos National Laboratory, Los Alamos, New Mexico \\ FRÉDÉRIC VIVIER \\ Laboratoire d'Océanographie et du Climat: Expérimentation et Approches Numériques, Paris, France \\ SARAH T. GiLle \\ Scripps Institution of Oceanography, La Jolla, California \\ Henk A. Dijkstra \\ Institute for Marine and Atmospheric Research Utrecht, Utrecht University, Utrecht, Netherlands
}

(Manuscript received 16 December 2005, in final form 11 December 2006)

\begin{abstract}
Observations of the sea surface height in the Argentine Basin indicate that strong variability occurs on a time scale of 20-30 days. The aim of this study is to determine the physical processes responsible for this variability. First, results are presented from two statistical techniques applied to a decade of altimetric data. A complex empirical orthogonal function (CEOF) analysis identifies the recently discovered dipole mode as the dominant mode of variability. A principal oscillation pattern (POP) analysis confirms the existence of this mode, which has a period of 25 days. The second CEOF displays a propagating pattern in the northern Argentine Basin, plus a rotating dipole in the southwest corner. The POP analysis identifies both patterns as individual modes, with periods of 30 and 20 days, respectively. Second, the barotropic normal modes of the Argentine Basin are studied, using a shallow-water model capturing the full bathymetry of the basin. Coherences between the spatial patterns of these modes and altimeter data suggest that several of the basin modes are involved in the observed variability. This analysis implies that the 20-day mode detected by recent bottom-pressure measurements is a true barotropic mode. However, the 25-day variability, as found in altimeter data, cannot be directly attributed to the excitation of a free Rossby basin mode. This study indicates that the results of several apparently conflicting observations of the flow variability in the Argentine Basin can be reconciled by assuming that multiple basin modes are involved.
\end{abstract}

\section{Introduction}

The circulation in the Argentine Basin exhibits extreme levels of variability. In altimeter data and model output alike (Fu and Smith 1996), the Argentine Basin is among the regions with the highest level of sea surface height (SSH) variability in the World Ocean, shar-

Corresponding author address: Dr. Wilbert Weijer, CCS-2, MS B296, Los Alamos National Laboratory, Los Alamos, NM 87545. E-mail: wilbert@lanl.gov ing the stage with well-known regions of high variability such as the Gulf Stream extension and the Agulhas retroflection.

A large part of this variability is related to the confluence of the highly energetic Malvinas and Brazil Currents, and the formation of the South Atlantic Current. This process gives rise to strong frontal motions and intense eddy activity (Garzoli and Simionato 1990; Goni and Wainer 2001; Vivier et al. 2001). In addition, there is evidence for a strong anticyclonic circulation around the Zapiola Rise, a sedimentary feature located at $45^{\circ} \mathrm{S}, 40^{\circ}-45^{\circ} \mathrm{W}$ (see Fig. 1). This circulation, esti-

DOI: $10.1175 / 2007 J P O 3527.1$

(C) 2007 American Meteorological Society 


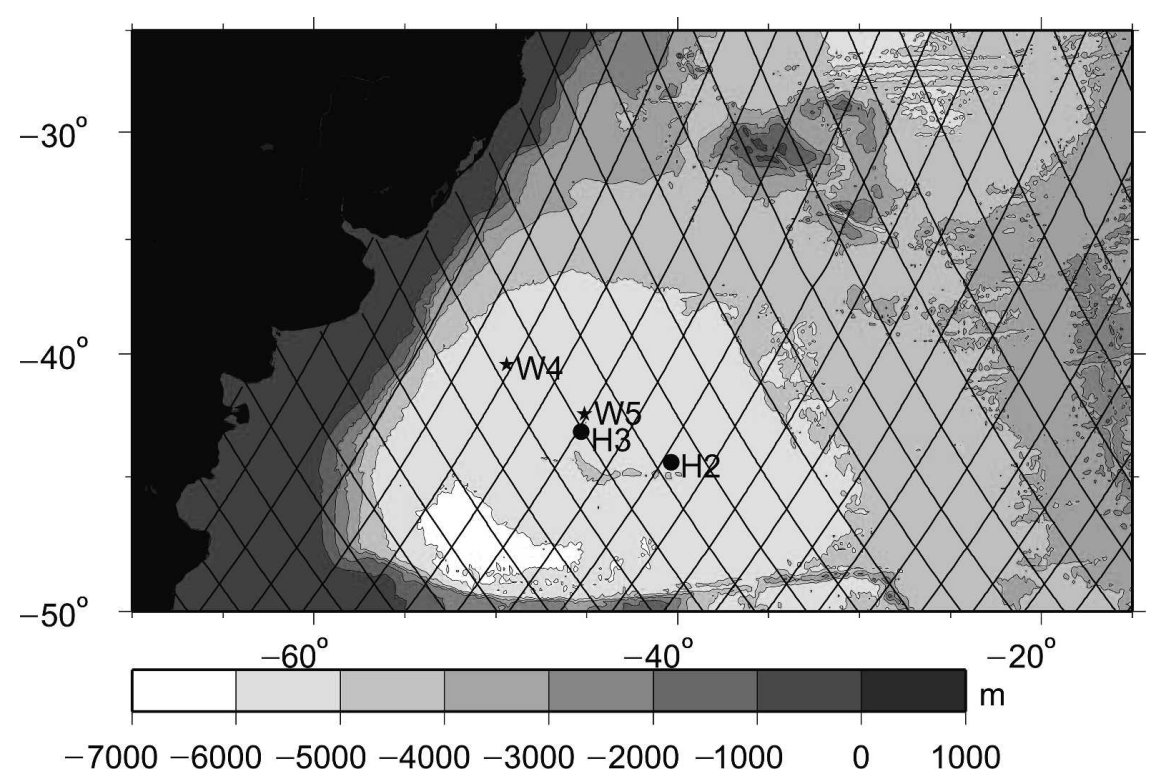

FIG. 1. Bathymetry of the Argentine Basin. Here W4 and W5 denote current-meter sites 4 and 5 of Weatherly (1993), whereas $\mathrm{H} 2$ and $\mathrm{H} 3$ are the positions of the bottom-pressure measurements of Hughes et al. (2007). Black lines are ground tracks of the TOPEX/Poseidon altimeter used by Fu et al. (2001).

mated to be in excess of $80 \mathrm{~Sv}\left(1 \mathrm{~Sv} \equiv 10^{6} \mathrm{~m}^{3} \mathrm{~s}^{-1}\right.$; Saunders and King 1995a), appears to be forced by eddies (Dewar 1998; De Miranda et al. 1999).

Several recent observations have revealed another source of variability. Fu et al. (2001, hereinafter FCQ) analyzed Ocean Topography Experiment (TOPEX)/ Poseidon altimeter data (collected along ground tracks shown in Fig. 1) for the 5-yr period between 1992 and 1997, and found a spectral peak at 25 days in SSH variability close to Zapiola Rise. After filtering the data to retain periods below 30 days, they identified a barotropic oscillation with a clear bipolar structure that appears to rotate around Zapiola Rise. They concluded that the oscillation must be a barotropic Rossby wave mode, trapped by the contours of $f / H$ surrounding the seamount (where $H$ denotes the water depth).

Barotropic variability in the Argentine Basin has been observed before. Weatherly (1993) analyzed current-meter records from a few moorings in the Argentine Basin and found evidence for barotropic variability with periods between 20 and 30 days. More specifically, he found evidence for a 28-day oscillation at the site closest to Zapiola Rise (his site 5; W5 in Fig. 1); at his site 4 (W4), roughly situated between Zapiola Rise and the continental shelf, he reported a spectral peak at 20 days.

Hughes et al. (2007) reported results from a yearlong deployment of two pressure gauges in the Argentine Basin (H2 and H3 in Fig. 1). They found a strong baro- tropic oscillation with a period of 19.6 days. These measurements suggest a transport variability associated with this mode of nearly $300 \mathrm{~Sv}$ (60 mbar). This makes it the most energetic barotropic mode identified in the World Ocean.

The discrepancy in the periods of these observations is remarkable, and a satisfactory explanation has not been found yet. Several factors have been suggested to explain the differences. Weatherly (1993) argued that the different peak frequencies at his sites 4 and 5 could be reconciled by accounting for a Doppler shift in the period of a single Rossby wave (with a basic period of about 23 days), due to the background flow being of opposite sign at the two locations. However, after correcting for a sign error (G. Weatherly 2005, personal communication), the direction of the flow at the two sites does not appear to support such an interpretation: accounting for a Doppler shift at site 5, the westward flow would actually be consistent with a free-oscillation period higher than 28 days, rather than lower. Similarly, the eastward flow at site 4 would increase the period of a free oscillation, up from a value below 20 days.

Alternatively, one might argue that the 20-day Nyquist period of the TOPEX/Poseidon data, as used by FCQ, is only marginally suited for detecting signals between 20 and 30 days. However, tests by FCQ and Tai and Fu (2005) show that it is the amplitude that is compromised by the marginal sampling, rather than the propagation characteristics. 
In this paper we propose that the flow variability in the Argentine Basin is caused by the excitation of several barotropic normal modes of this basin. The theoretical basis for this hypothesis is the fact that closed basins with idealized geometry exhibit a discrete spectrum of eigenmodes, also known as Rossby basin modes (Longuet-Higgins 1964). These basin modes are superpositions of Rossby waves that satisfy the boundary conditions of a basin. In a companion paper (Weijer et al. 2007, hereinafter Part II) we demonstrate that the classical spectrum carries over to a realistic basin, with the modes being deformed by bathymetry. The excitation of multiple oscillatory Rossby basin modes would reconcile the apparently conflicting observational results on the frequency of flow variability in the Argentine Basin.

In section 2, results will be presented for two statistical techniques applied to a decade of altimeter data. These analyses reveal the presence of statistical modes with periods of about 20,25, and 30 days, and support the hypothesis of multiple oscillatory modes. In section 3 , the spectrum of barotropic normal modes of the Argentine Basin is presented, using a shallow-water model. This spectrum reveals several dynamical modes with periods in the 20-30-day range.

Schnur et al. (1993) pioneered the comparison of principal oscillation patterns (POPs) with eigenmodes from a theoretical model and concluded that "the most significant POPs are very similar in time and spatial structure to the most unstable waves in the stability analysis" of their quasigeostrophic model. In this study we go a step further by using dynamical modes to extract information from the observations: the time series obtained by projecting their spatial structures on altimeter data yields significant signals that suggest the presence of several modes in the observations (section 4). This technique can be useful for finding barotropic modes in noisy data, especially when their periods, and in some cases their spatial structures, are alike. In section 5, we study the excitation of the dynamical modes in the shallow-water model and their impact on the variability of the flow. Section 6 discusses the results and addresses the origin of the 20- and 25-day variability found in observations.

\section{Multiple statistical modes in altimeter data}

In this section we present a detailed statistical analysis of weekly maps of SSH for the period 1992-2002. The data represent merged TOPEX/Poseidon (T/P) and European Remote Sensing Satellite (ERS) altimeter observations, mapped onto a $1 / 3^{\circ}$ Mercator grid, and provided by Archiving, Validation, and Interpretation of Satellite Oceanographic (AVISO) data (Ducet et al. 2000). The data have been box-averaged to a $2^{\circ}$ spatial resolution to reduce the signature of eddies, and have subsequently been high-pass-filtered in time to retain periods shorter than 35 days. The spatial average has been removed from each map to emphasize shorter spatial scales. For the POP analysis only, the data are subjected to an empirical orthogonal function (EOF) truncation, in order to further reduce noise levels while retaining the dominant modes of variability (Von Storch and Zwiers 1999, p. 338). The first 35 EOFs are retained, which altogether account for $92 \%$ of the SSH variance. For comparison, FCQ mapped T/P data on a $1^{\circ}$ grid using a Gaussian-weighted smoothing scheme. They chose a Gaussian weight with half-widths of $1^{\circ}$ latitude $\times 2^{\circ}$ longitude. They filtered the data to retain periods below 30 days.

The merged T/P and ERS data product has a higher temporal resolution (nominally 7 days) than that of T/P data alone (10 days). The AVISO data hence can be expected fully to capture the signals of interest with periods between 20 and 30 days.

\section{a. Complex empirical orthogonal functions}

First, these data have been subjected to a complex EOF (CEOF) analysis. This statistical technique searches for orthogonal patterns that optimize the amount of variance. Expanding the principal time series $\mathbf{X}$ with its time-derivative $\mathbf{X}_{t}$ (Hilbert transform) allows for detecting propagating signals. CEOFs are by construction orthogonal, and are purely statistical objects: in contrast to POP analysis, no a priori model is assumed.

Applied to the weekly SSH data, the two dominant modes, CEOF 1 and CEOF 2, account for $32.8 \%$ and $14.4 \%$ of the variance of the filtered data, respectively. Their spatial patterns are shown in Figs. 2a and 2b. The next largest mode accounts for only $7 \%$ of the variance, so CEOFs 1 and 2 are clearly the dominant statistical patterns. To put this in perspective, CEOF 1 explains only $1.4 \%$ of the variance of the unfiltered dataset (still on the $2^{\circ}$ grid), CEOF 2 only $0.6 \%$. This is a direct consequence of the ubiquitous "redness" of oceanic spectra, which assigns most of the energy to the lowfrequency variability (e.g., Wunsch and Stammer 1995).

The power spectral densities (PSD) of the real expansion coefficient of CEOF 1 and CEOF 2 are shown in variance-preserving form (PSD $\times$ frequency) in Fig. 2c. They were estimated using the adaptive form of the multitaper method (Thomson 1982) using a timebandwidth product parameter of 4. Circles in Fig. 2c 
a)

CEOF 1: $32.8 \%$ variance
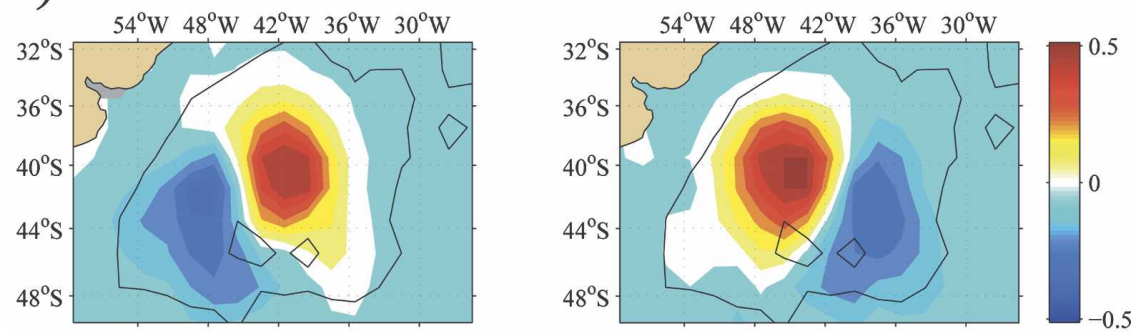

b)

CEOF 2: $14.4 \%$ variance

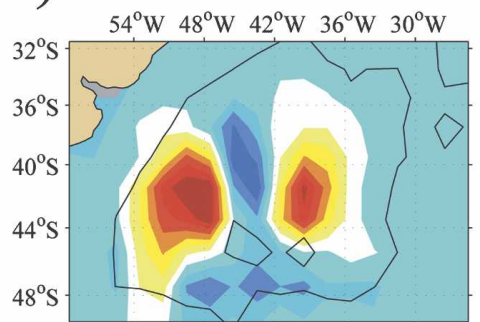

Real pattern

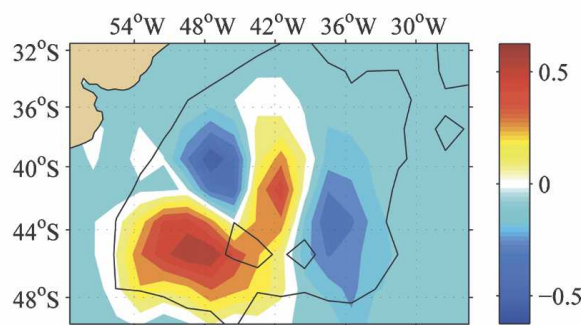

minus Imaginary pattern

c)

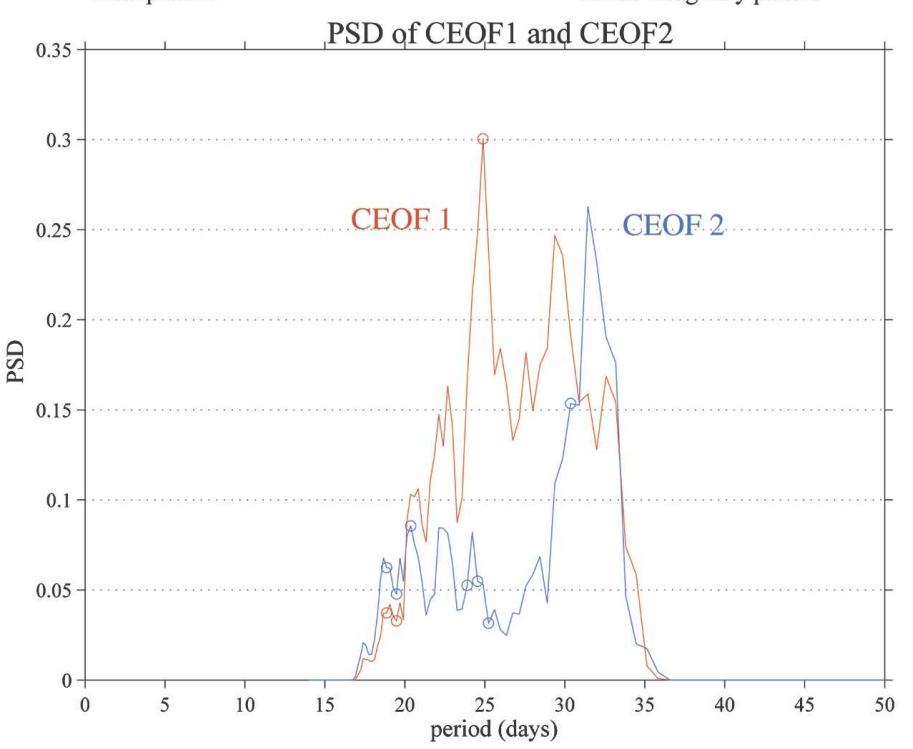

FIG. 2. CEOF analysis of altimeter data in the Argentine Basin. Shown are the (left) real and (right) minus imaginary spatial patterns of (a) the first and (b) the second CEOF. Together, they account for $47.2 \%$ of the variance. The modes evolve as $\mathfrak{R}(\mathbf{p}) \rightarrow-\mathfrak{I}(\mathbf{p}) \rightarrow-\mathfrak{R}(\mathbf{p}) \rightarrow$ $\mathfrak{I}(\mathbf{p}) \rightarrow \Re(\mathbf{p})$. The black line denotes $f / H=-2 \times 10^{-8} \mathrm{~m}^{-1} \mathrm{~s}^{-1}$. (c) The power spectral densities (in variance-preserving form) of the corresponding real expansion coefficients. Circles denote a positive identification of a harmonic signal at the $93 \%$ level, based on the method of Thomson (1982).

indicate periodic components that are statistically significant relative to colored noise, based on an $F$ test with a confidence level of $93 \%$ (Thomson 1982).

CEOF 1 (Fig. 2a) clearly reflects the bipolar structure first identified by FCQ. The spectral characteristics of its expansion coefficient (Fig. 2c) show a spectral peak at the period of 25 days, which is identified as a harmonic signal at the $93 \%$ confidence level. In addition, there is a secondary peak just below 30 days. As will become clear after comparison with the POPs as presented in the next section, CEOF 2 (Fig. 2b) seems to combine two signals: a plain wave propagating westward in the northern part of the Argentine Basin, plus a rotational mode in the southwestern corner. The 
spectral characteristics of its expansion coefficient (Fig. 2c) reveal a maximum at a period of 31 days, plus a series of secondary maxima around 20 days. The peak at 20 days is identified as a harmonic signal at the $93 \%$ confidence level. The signal at 31 days might not be perfectly harmonic.

\section{b. Principal oscillation patterns}

The second technique to diagnose the space-time variability of the Argentine Basin is the POP analysis (Hasselmann 1988; Von Storch et al. 1988). This technique is designed to extract statistical modes of variability from a noisy dataset, based on the assumption that the variability is governed by a stochastic firstorder autoregressive (AR-1) process. In contrast to the CEOF method, POP analysis does not search for patterns that optimize the amount of variance. This relieves the modes from the burden of orthogonality, so that POPs may be better suited than CEOFs to describe the variability of a nonnormal system.

An AR-1 process assumes the state vector $\mathbf{X}$ to obey a stochastic difference equation of the form

$$
\mathbf{X}_{t+1}=\mathbf{A} \mathbf{X}_{t}+\text { noise. }
$$

In POP analysis, $\mathbf{A}$ is estimated as $\mathbf{A}=$ $\left\langle\mathbf{X}_{t+1} \mathbf{X}_{t}^{\mathrm{T}}\right\rangle\left\langle\mathbf{X}_{t} \mathbf{X}_{t}^{\mathrm{T}}\right\rangle^{-1}$. The POPs $\mathbf{p}_{k}$ are the normalized eigenmodes of $\mathbf{A}$, with eigenvalues $\lambda_{k}$. Because $\mathbf{A}$ is real but generally asymmetric, POPs are real or occur in complex conjugate pairs. The period of a POP is given by $2 \pi /\left|\arg \left(\lambda_{k}\right)\right|$, and its damping time scale is $-1 /$ $\log \left(\left|\lambda_{k}\right|\right)$. The evolution of each POP over a period is given by the real (denoted by $\mathfrak{R}$ ) and imaginary $(\mathfrak{I})$ part of $\mathbf{p}_{k}$ following the sequence

$$
\mathfrak{R}\left(\mathbf{p}_{k}\right) \rightarrow-\mathfrak{I}\left(\mathbf{p}_{k}\right) \rightarrow \mathfrak{R}\left(\mathbf{p}_{k}\right) \rightarrow \mathfrak{I}\left(\mathbf{p}_{k}\right) \rightarrow \mathfrak{R}\left(\mathbf{p}_{k}\right) .
$$

The three dominant modes of this analysis, as applied to the weekly SSH data, are presented in Fig. 3. The dominant mode, POP 1, displays a plane-wave propagation in the northern part of the Argentine Basin. Its period is 30 days. POP 2, on the other hand, at 20 days, displays a clear bipolar pattern, situated in the western part of the basin. A similar structure, albeit with larger lobes and positioned more centrally, is displayed by POP 3. Its period is 25 days.

To test the robustness of the results, we performed a POP analysis on each of the two halves of the original time series. We found the results to be consistent with those of the full time series. Furthermore, we consistently found a high and significant coherence between the real and imaginary time series of each POP at its associated period, and found them to be consistently $90^{\circ}$ out of phase. This suggests that the POPs are physically meaningful (Von Storch et al. 1988).
A comparison of the POPs in Fig. 3 and the CEOFs of Fig. 2 suggests that both methods capture the same modes. From its peak spectral power at 25 days, as well as from its bipolar, rotating pattern, it is clear that CEOF 1 is dominated by POP 3 . CEOF 2 , on the other hand, shows the clear signatures of both POPs 1 and 2; not only does CEOF 2 capture the spatial patterns of both modes, but the spectrum of the expansion coefficients clearly features the spectral peaks at 20 and 30 days.

\section{Barotropic Rossby basin modes of the Argentine Basin}

In this section, we determine the barotropic basin modes of the Argentine Basin by solving the linear stability problem of the motionless state in a barotropic shallow-water model. This model is based on the code used by Schmeits and Dijkstra (2000), but includes full bathymetry and bottom friction. The numerical grid has a spatial resolution of $0.5^{\circ} \times 0.25^{\circ}$, and covers the region from $25^{\circ}$ to $50^{\circ} \mathrm{S}$ and $15^{\circ}$ to $69^{\circ} \mathrm{W}$. Bathymetry is based on 2-minute gridded elevations/bathymetry for the world (ETOPO2) dataset. Depths shallower than $300 \mathrm{~m}$ (the continental shelf) are set to zero. The eigenvalue problem is solved using the Jacobi-Davidson "QZ" method (JDQZ; Sleijpen and Van der Vorst 1996). Horizontal viscosity $A_{h}$ and bottom friction $v$ are set to $3.0 \times 10^{3} \mathrm{~m}^{2} \mathrm{~s}^{-1}$ and $3.2 \mathrm{day}^{-1}$, respectively. For further details of the model and the solution procedure we refer to Part II of this study.

The seven eigenmodes having oscillation periods smaller than 50 days are shown in Fig. 4. Modes 1-4 all display rotating multipole structures, with oscillation periods of 19.6, 22.7, 27.7, and 31.5 days, respectively. Modes 1, 3, and 4 are characterized by a bipolar pattern, which is either large-scale (modes 1 and 4) or small-scale (mode 3 ). Mode 2 features a quadrupole structure. Modes 5, A, and 6 (with periods of 39.7, 41.6, and 45.2 days) are largely confined to the eastern part of the Argentine Basin. Animations show that these modes are characterized by the southward propagation of anomalies. (Note that the oscillatory periods are determined with high numerical accuracy, but do depend on details of the model formulation. As is shown in Part II, uncertainties in especially horizontal viscosity may affect the oscillation time scales by about $5 \%$.)

In the next section we will use the spatial structure of the basin modes, as predicted by the shallow-water model, to find evidence that some of these modes are actually excited. To that end, we project the eigenmodes on altimeter data, and analyze the resulting time series for significant spectral signals. 
a) POP 1 : Period= $30.0 \mathrm{~d}$; damp: $126.3 \mathrm{~d}-$ variance $\%: 14.3$
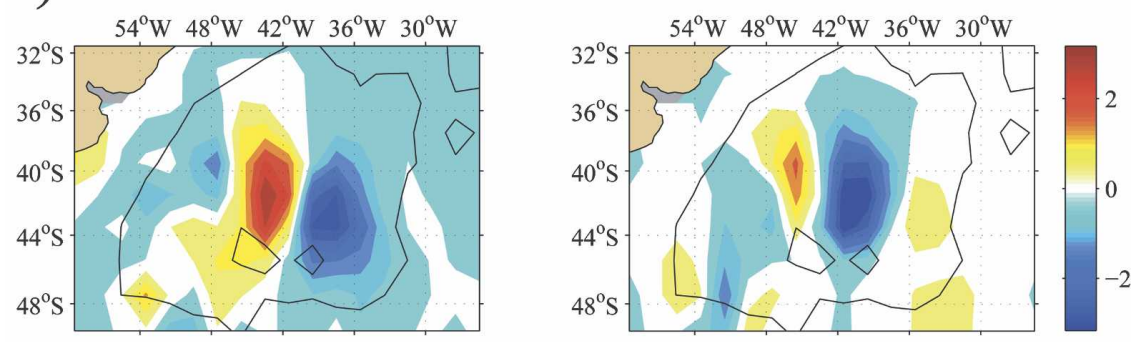

b)
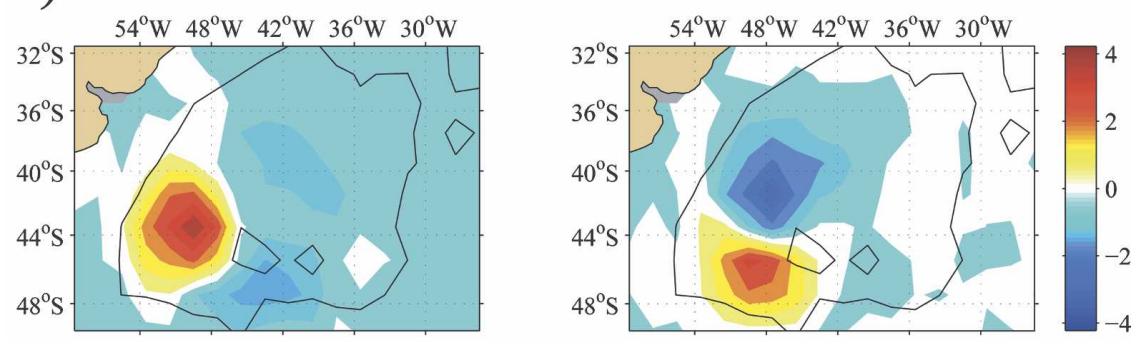

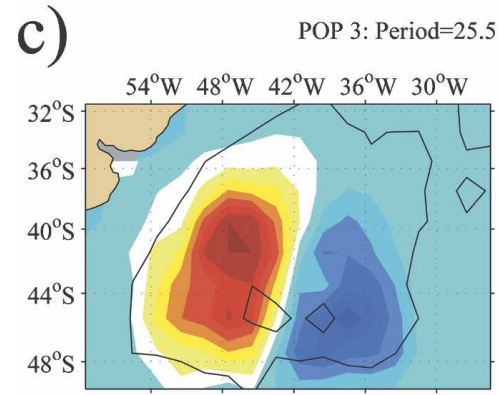

Real pattern

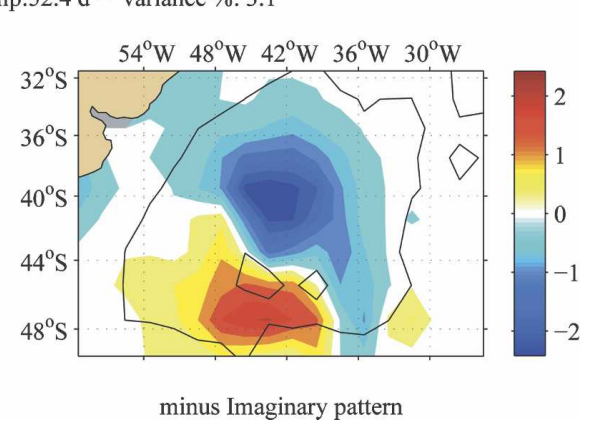

FIG. 3. POP modes from altimeter SSH. Mode with a period of (a) 30, (b) 20.8, and

(c) 25.5 days. The black line denotes $f / H=-2 \times 10^{-8} \mathrm{~m}^{-1} \mathrm{~s}^{-1}$.

\section{Combining dynamical modes and observations}

We use altimeter data as provided by AVISO for the period between 14 October 1992 and 5 January 2005. This yields $N=638$ weekly snapshots of the sea surface height. The data, provided on a $13^{\circ}$ Mercator grid, are interpolated onto our model grid, and the mean value is subtracted for every snapshot. The normal modes of our shallow-water mode (Fig. 4) are normalized, yielding a set of $M=14$ spatial patterns $\left[\psi_{i}^{R}(\mathbf{x}), \psi_{i}^{I}(\mathbf{x})\right]$, $i=1, \ldots, 7$. For the moment we will drop the superscripts $R$ and $I$ and ignore the fact that the patterns come in pairs. We assume that the data $S(\mathbf{x}, t)$ consist of a superposition of the modes and noise $Z$ :

$$
S(\mathbf{x}, t)=\sum_{i=1}^{M} \psi_{i}(\mathbf{x}) \gamma_{i}(t)+Z(\mathbf{x}, t) .
$$

Multiplying this equation by each of the modes $\psi_{j}$ and integrating over the domain $D$ yields

$$
\int_{D} \psi_{j} S d x d y=\sum_{i=1}^{M} \mathbf{C}_{j i} \gamma_{i}+\int_{D} \psi_{j} Z d x d y
$$

with

$$
\mathbf{C}_{j i}=\int_{D} \psi_{j} \psi_{i} d x d y
$$

The last integral in Eq. (3) vanishes since it is assumed that the noise is spatially uncorrelated to the eigenmodes. The matrix $\mathbf{C}$ gives the correlations between the eigenmodes; if the set were orthonormal, $\mathbf{C}$ would equal the identity matrix $\mathbf{I}$, but it is not. Therefore, a full matrix inversion is performed at each time step to find the unique set of 14 time series $\gamma_{i}(t)$, which will be grouped in pairs $\left[\gamma_{i}^{R}(t), \gamma_{i}^{I}(t)\right], i=1, \ldots, 7$.

If a mode explains part of the variability in the data, there should be a significant coherence between $S(\mathbf{x}, t)$ and the reconstruction $\hat{S}_{i}=\gamma_{i}^{R}(t) \psi_{i}^{R}(\mathbf{x})+\gamma_{i}^{I}(t) \psi_{i}^{I}(\mathbf{x})$ in 
a) Mode 1
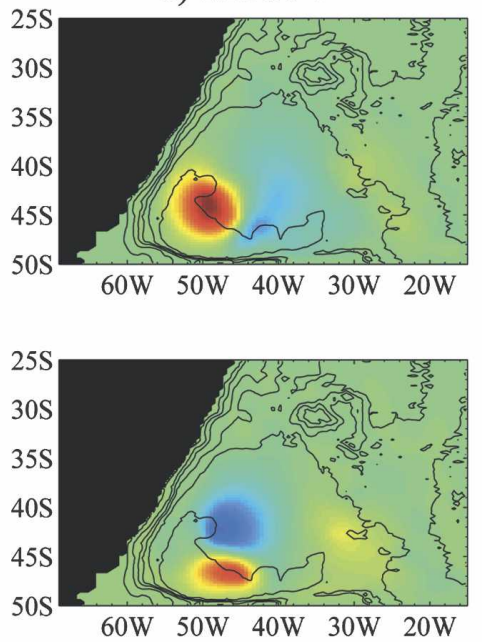

c) Mode 3
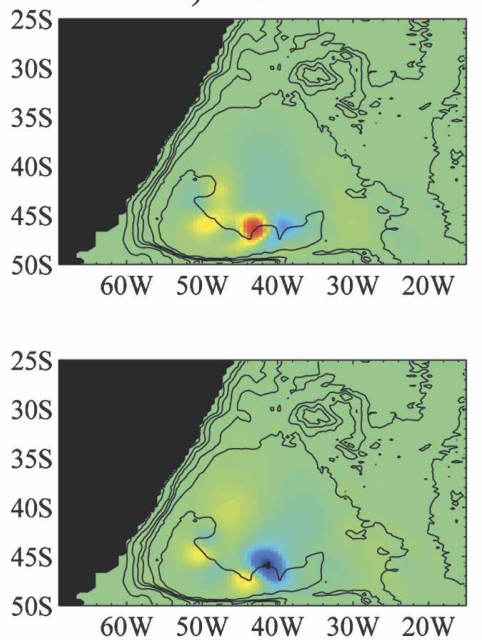

b) Mode 2
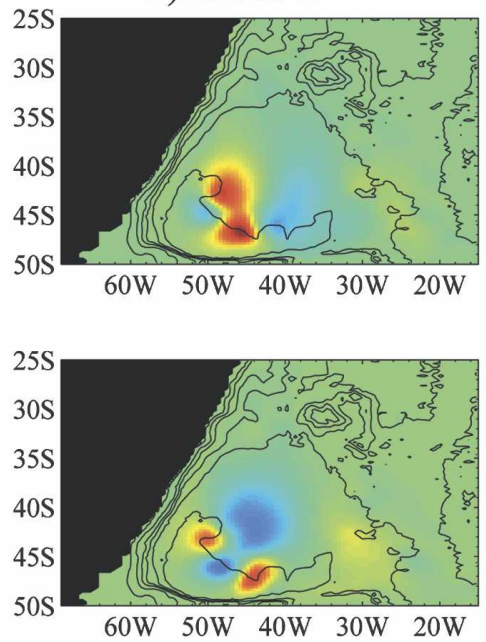

d) Mode 4
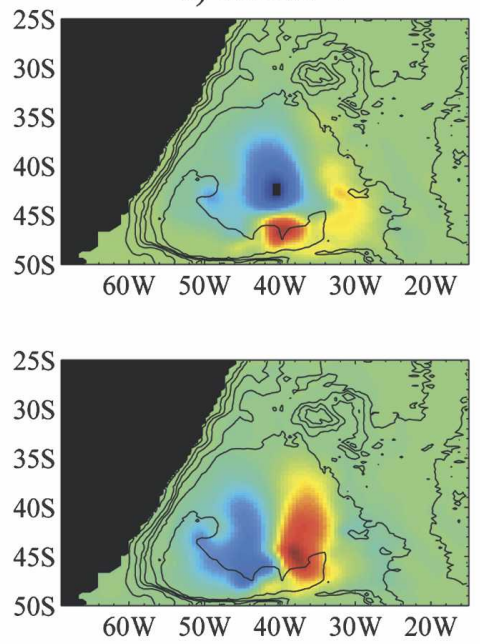

e) Mode 5
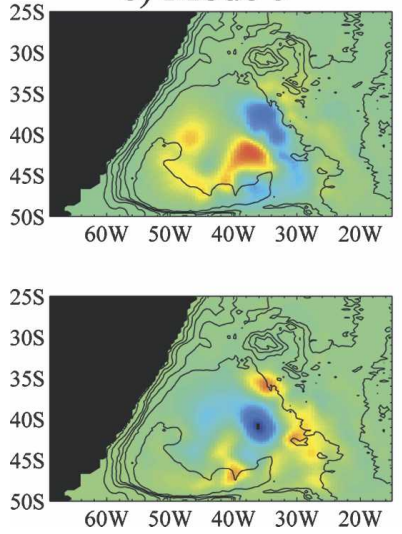

f) Mode A
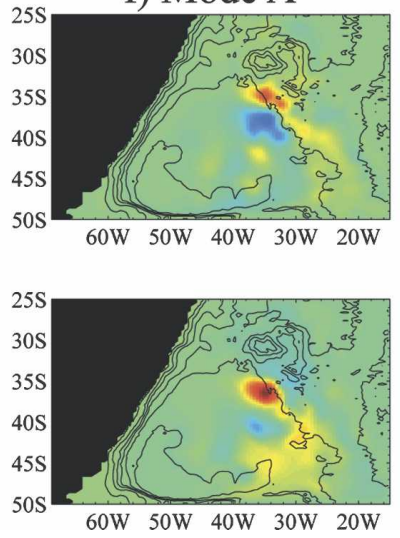

g) Mode 6
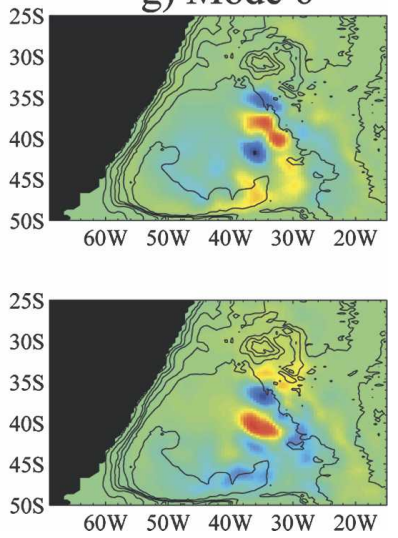

FIG. 4. Spatial patterns of the first seven eigenmodes of a barotropic shallow-water model, configured for the Argentine Basin. The upper plots in each panel show real parts of the basin modes; lower plots show the imaginary parts. (a) Mode 1 (with a period of 19.6 days), (b) mode 2 (22.7 days), (c) mode 3 (27.7 days), (d) mode 4 (31.5 days), (e) mode 5 (39.7), (f) mode A (41.6 days), and (g) mode 6 (45.2 days). Units are arbitrary; the color scale ranges from blue $(-\max |\eta|)$ to red $(\max |\eta|)$, and centers around green $(0)$. 


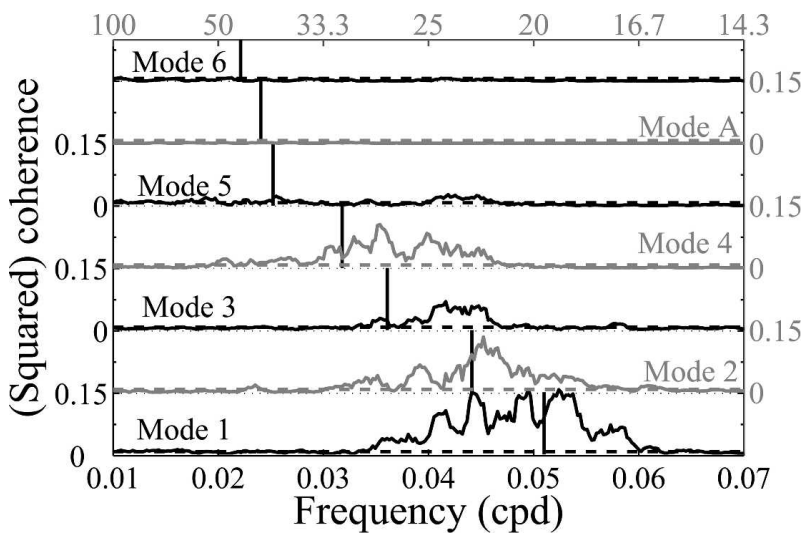

FIG. 5. Coherence analysis between the altimeter dataset $S$ and the reconstructions $\hat{S}_{i}$. The curves for each of the seven normal modes are alternately colored black and gray, and are referenced to the left and right ordinate, respectively. Dashed lines denote 99\% significance level (0.0066) as determined from a simple estimate of the number of degrees of freedom. Vertical lines indicate frequency as determined from the normal-mode analysis.

a frequency band near the oscillation frequency. To test this, we calculated the coherence between $S$ and $\hat{S}_{i}$ over the entire domain, for each of the seven eigenmodes, $i=1-7$. Following Von Storch and Zwiers (1999), the square-coherency spectrum is defined here as

$$
\kappa_{x y}(\omega)=\frac{\left|\Gamma_{x y}(\omega)\right|^{2}}{\Gamma_{x x}(\omega) \Gamma_{y y}(\omega)},
$$

where $\Gamma_{x x}$ and $\Gamma_{y y}$ are the power spectra of the processes $x$ and $y$, and $\left|\Gamma_{x y}\right|$ is the amplitude of their crossspectrum.

For each of the 7534 (ocean) grid points, the crossspectrum is calculated, and a composite cross-spectrum is obtained by averaging over geographic locations. The data do not represent 7534 independent records because of the large spatial coherence of the variability. Using strategies suggested by Bretherton et al. (1999), we estimated the effective spatial degrees of freedom of the AVISO data to be about 100 . To reduce the noise, we smoothed the spatial average by taking the 7-point moving average of the spectral components (Daniell filter; Von Storch and Zwiers 1999). The resulting spectra are equivalent to spectra computed using roughly 700 independent data segments (and thus have 1400 degrees of freedom for coherence calculations). In this case $\kappa_{x y}(\omega)$ is significantly different from zero at the $99 \%$ level if it exceeds 0.0066 . As a reality check, we also computed significance levels using a Monte Carlo method to randomly scramble the data records, shuffling them in time while retaining the spatial structure. This produced comparable significance estimates.

Figure 5 shows the result of this coherence analysis,

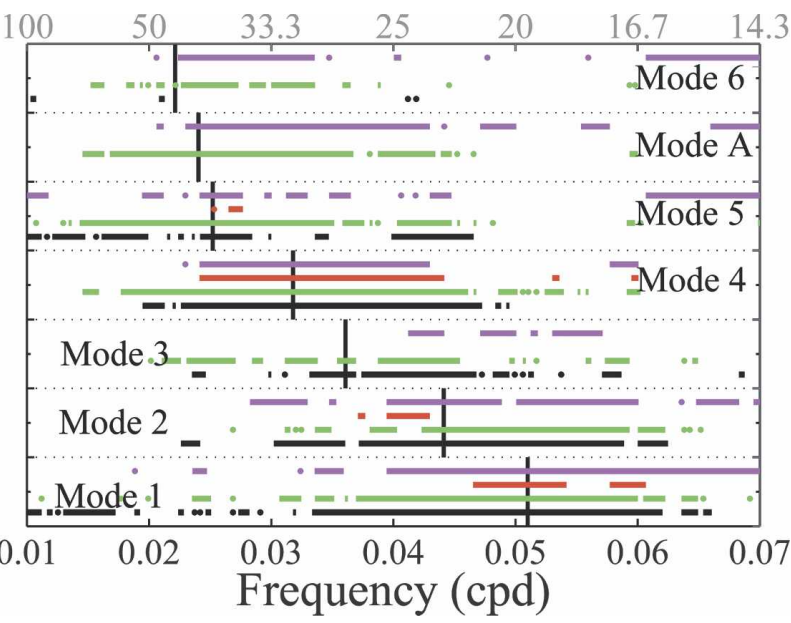

FIG. 6. Significance windows for the coherence analyses as described in the text. For each of the seven modes, the colored bars indicate the frequencies where a coherence exceeds the $99 \%$ significance level. Black and green lines represent altimeter data; red and magenta represent the model output. Coherences are calculated between the data $S$ and reconstructions $\hat{S}_{i}$ (black, red), and between the real $\left(\gamma_{i}^{R}\right)$ and imaginary $\left(\gamma_{i}^{I}\right)$ time series of each mode (green, magenta).

with the solid lines representing the coherence of the signal, while the dashed lines denote the $99 \%$ significance level. The black lines in Fig. 6 indicate the frequency bands where this significance level is achieved. Other significance tests, discussed later, are represented by the lines of different color. The vertical bars indicate the modal eigenperiods.

These results indicate that a number of modes are indeed significantly present in the data. This is particularly true for modes $1-4$. Mode 5 also exceeds the significance level for a broad frequency range, but its values are only marginally significant. Modes A and 6 appear to be nowhere significant. It is reassuring that the frequency range where significant values are found seems to shift to lower frequencies for increasing mode number. This is consistent with the increase in the corresponding eigenperiod.

Mode 1 shows the largest coherences. It seems to be significant for periods between 15 and 30 days. The curve displays several pronounced peaks, most specifically at periods of 19, 20,23, and 24 days. Its theoretical period, as determined from the normal-mode analysis, is 19.6 days. Mode 2 is significant for periods between 18 and 33 days, mode 3 between 21 and 31 days, while mode 4 spans the range between 21 and 50 days.

Repeating the analysis for several configurations, doing an ad hoc projection (i.e., taking the matrix $\mathbf{C}=\mathbf{I}$ ), or orthogonalizing the eigenmodes using the GramSchmidt algorithm, shows that the results are qualitatively robust. Furthermore, when the reconstructions $\hat{S}_{i}$ 
are based on each of the patterns individually, instead of the combination of $\psi_{i}^{R}$ and $\psi_{i}^{I}$ for each eigenmode, the coherences between the reconstructions and the data $S$ are very similar for the two members of a single eigenmode, and closely resemble the original results of Fig. 5. Also applying another spectral estimator by segmenting the dataset into shorter sections, and averaging the resulting spectra (chunk spectral estimator; Von Storch and Zwiers 1999), yields comparable results. This spectral estimator also confirmed that the statistically significant spectral peaks do not vary substantially when shorter temporal subsets of the record are used, implying that the modal response is more or less stationary in time.

When an oscillatory mode is present, the time series of the real and imaginary parts of each mode should be coherent and about $90^{\circ}$ out of phase. To test this, the coherence was calculated between $\gamma_{i}^{R}(t)$ and $\gamma_{i}^{I}(t)$ for each mode using a 7-point Daniell filter as above. The green lines in Fig. 6 show where the coherence is significant above the $99 \%$ level. The green lines of modes 1 to 5 cover roughly the same frequency ranges as the black lines. The phases are stable for these significance regions and are not significantly different from $-90^{\circ}$ : their average and standard deviation over the significant regions are $-94 \pm 13,-80 \pm 13,91 \pm 18,-100 \pm$ 11 , and $-100 \pm 16$ for modes 1 to 5 , respectively. Interestingly, the phase relation for mode 3 is positive, suggesting a cyclonic motion instead of the anticyclonic rotation implied by the dynamic mode. The reason for this is unclear but is possibly related to the fact that mode 3 has the smallest spatial structure of all the modes determined, and is likely to be captured least accurately by the altimeter and the gridding procedure used in the postprocessing.

\section{Excitation of Rossby basin modes in the shallow-water model}

The normal-mode analysis in section 3 has shown that the shallow-water model does not contain a Rossby basin mode with a 25-day time scale and bipolar structure similar to the mode found by FCQ. This, however, does not exclude the possibility that a forced model could experience 25 -day periodicity.

To test its response to forcing, we integrated the model for 1703 days, from August 1999 until March 2004. Wind forcing is based on a $0.5^{\circ} \times 0.5^{\circ}$ blended product, which combines Quick Scatterometer (QuikSCAT) data and National Centers for Environmental Prediction reanalysis wind fields (available online at: dss.ucar.edu/datasets/ds744.4/; Milliff et al. 2004). Daily averages are constructed by averaging the 6-hourly data; daily values are linearly interpolated during the day. Three-day averaged fields of SSH are output and used for diagnostics. Bottom friction is reduced to $r=0.0157$ day $^{-1}$ to increase the damping time scale of the eigenmodes, while $A_{h}$ is kept at its standard value. An implicit (Crank-Nicholson) time-stepping scheme is applied, with a time step of $20 \mathrm{~min}$.

The three-daily SSH fields are subjected to POP and CEOF analyses. First, the time series are bandpassfiltered for periods between 17 and 35 days. Then, for the POP analysis only, a 23 EOF prefiltering is applied that retains over $99 \%$ of the variance.

Figure 7 shows the four dominant POP modes, the first two capturing more than $15 \%$ of the variance each. Comparison of the spatial patterns and oscillation periods suggest that POPs 1 and 4 represent the free dynamical modes 1 and 4 (Figs. 4a,d). The oscillation periods (20.5 days for POP 1 versus 19.6 days for normal mode 1; 28.6 days for POP 4 versus 31.5 days for normal mode 4) are not inconsistent with this interpretation, especially considering their range of influence as inferred from the coherence analysis (Fig. 6). POPs 2 and 3 , however, cannot readily be associated with free dynamical modes.

Figure 8 shows the dominant CEOFs and the power spectral densities (PSD) of their (real) time series. Clearly, CEOF 1 accounts for most of the variance $(62.6 \%)$. It displays a large-scale structure that appears to alternate between a dipole (real pattern) and a monopole (imaginary pattern). Its spectral power is concentrated between 20 and 25 days, and peaks at 21.4 and 23.3 days. These peaks are close to the oscillation periods of POPs 3 (21.9 days) and 2 (23.8 days); this suggests that these statistical modes contribute to CEOF 1, although there is no obvious correspondence between the spatial patterns of CEOF 1, and POPs 2 and 3 . CEOF 2 is much less energetic, capturing only $13.8 \%$ of the variance. The spatial patterns essentially reflect a dipole structure rotating in the Zapiola Rise region. Its PSD has spectral peaks at 19.2 and 22.6 days. These peaks are close to the oscillation periods of dynamical modes 1 (19.6 days) and 2 (22.7 days).

This analysis shows that the model response to wind stress forcing is dominated by variability that has no obvious connections to the known free barotropic modes, and whose energy is concentrated in the frequency band between 20 and 25 days. Although the excitation of the free modes is evident from the POP analysis, other mechanisms appear to be in play that are responsible for the bulk of the variance.

\section{Discussion}

The results presented in this paper suggest that the variability in the Argentine Basin is at least partly gov- 


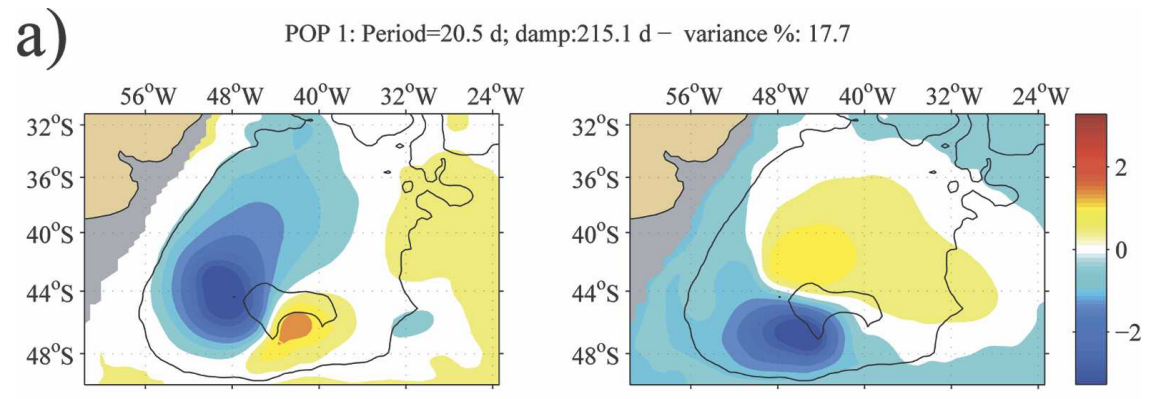

b) POP 2: Period=23.8 d; damp:58.2 d - variance \%: 15.5

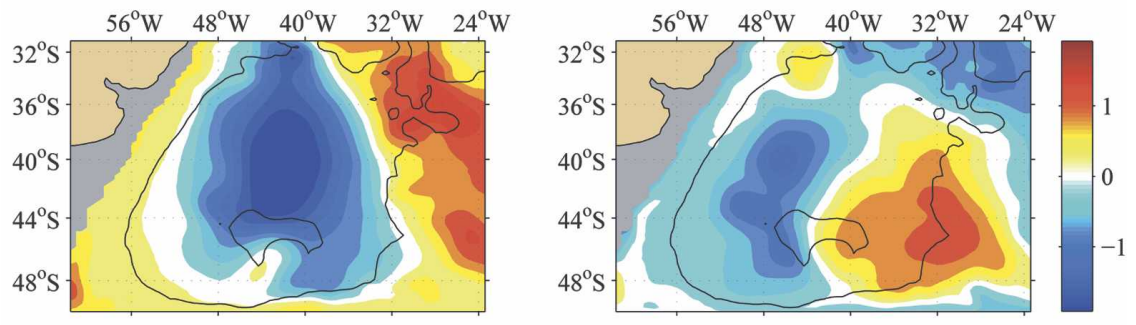

C) POP 3: Period=21.9 d; damp:71.9 d - variance \%: 9.6

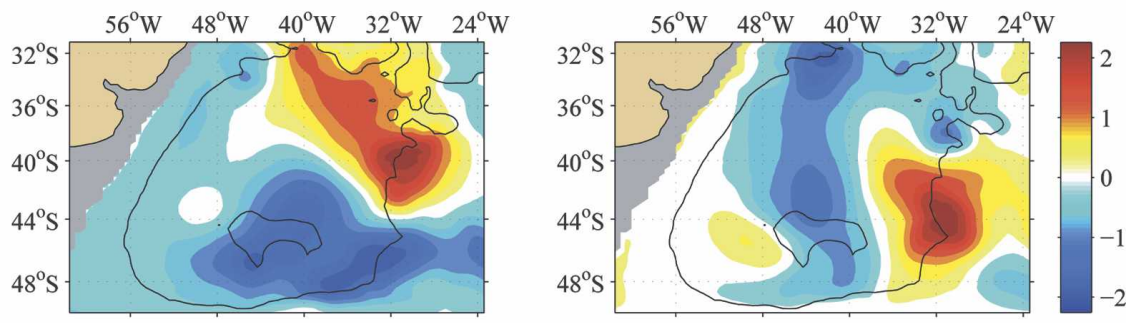

d) POP 4: Period=28.6 d; damp:75.9 d - variance \%: 8.1

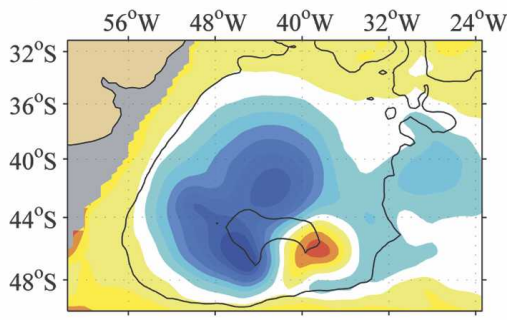

Real pattern

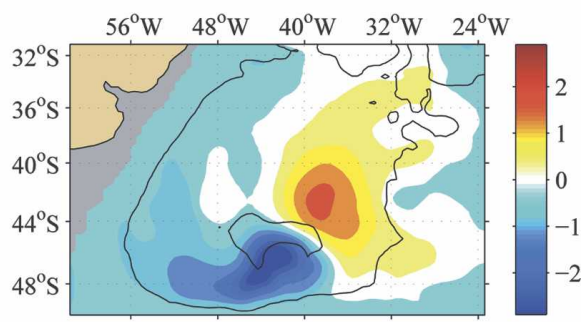

minus Imaginary pattern

FIG. 7. First four POP modes from model SSH.

erned by the excitation of a spectrum of free Rossby basin modes. The most compelling case can be made for a rotating mode with a period of about 20 days. Found as the dominant signal in the bottom-pressure data of Hughes et al. (2007), as well as in the current-meter data of Weatherly (1993), it turned up consistently in our analyses of weekly altimeter data. These observations are consistent in period and spatial structure with the dominant mode of our normal-mode analysis. Hence, this 20-day mode can be interpreted as the fun- damental barotropic basin mode of the Argentine Basin. In addition, the time integration of our model shows that it is excitable by wind stress alone, although other excitation mechanisms (like eddy forcing) may play a role as well.

In addition to this 20-day mode, normal-mode analysis of the Argentine Basin suggests the presence of another mode with a similar bipolar structure, but with a lower rotation frequency of once every 31.5 days (Fig. 4d). A POP analysis performed on the AVISO altim- 
a) $\quad$ CEOF 1: $62.6 \%$ variance
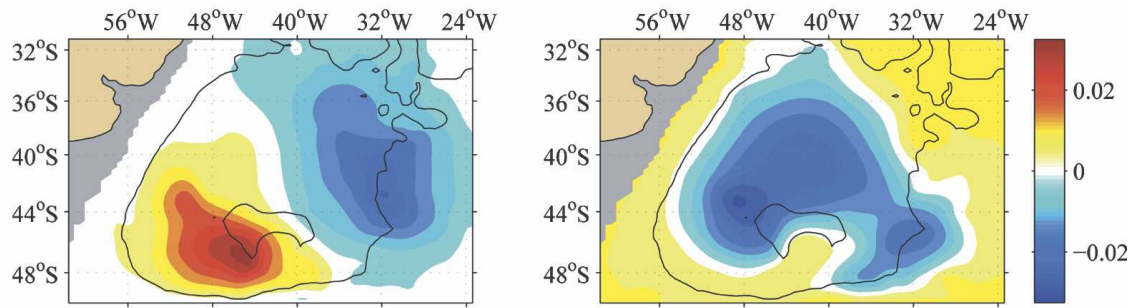

\section{b)}

CEOF 2: $13.8 \%$ variance

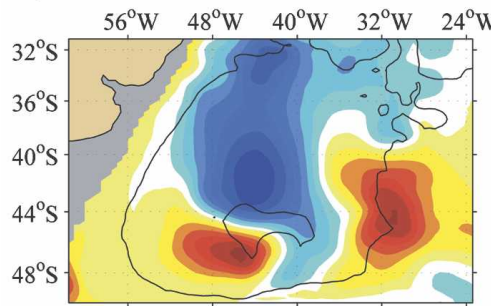

c)

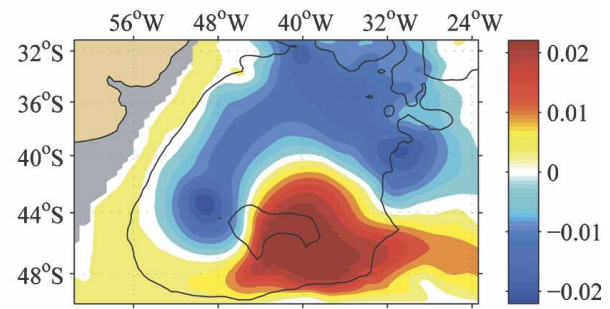

minus Imaginary pattern

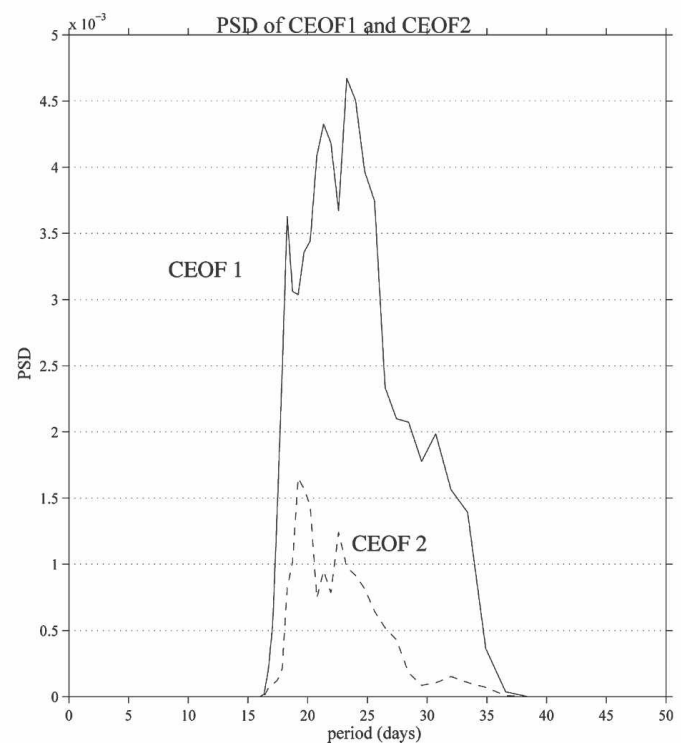

FIG. 8. Dominant CEOF modes from model SSH. (a) The first CEOF, accounting for $62.6 \%$ of the variance, and (b) the second CEOF, $13.8 \%$. (c) The power spectral densities (in variance-preserving form) of the corresponding real expansion coefficients.

eter data (Fig. 9), but now filtered for the period range between 30 and 33 days, indeed detects a statistical mode with a period that is consistent with this normal mode 4 in both period and spatial pattern (Fig. 9b). Its center of rotation is offset about $5^{\circ}$ east with respect to that of mode 1 . This mode could be responsible for the spectral peak at 28 days in the current-meter records at site 5 of Weatherly (1993).

Our CEOF analysis of AVISO altimeter data reproduces the dominance of the 25-day variability, as found by FCQ in TOPEX/Poseidon data. In addition, its emergence as a distinct statistical mode in the POP analysis reinforces the suggestion that a dynamical mechanism may underlie its existence. However, we have been unable to link this feature unambiguously to a free basin mode, since the normal-mode analysis did not turn up a mode that is consistent with these observations. How, then, can this feature be explained?

One way to reconcile the discrepancy between the observations and our normal-mode analysis is to as- 
a) POP 1: Period $=32.3 \mathrm{~d}$; damp:44.3 d - variance $\%: 19.9$
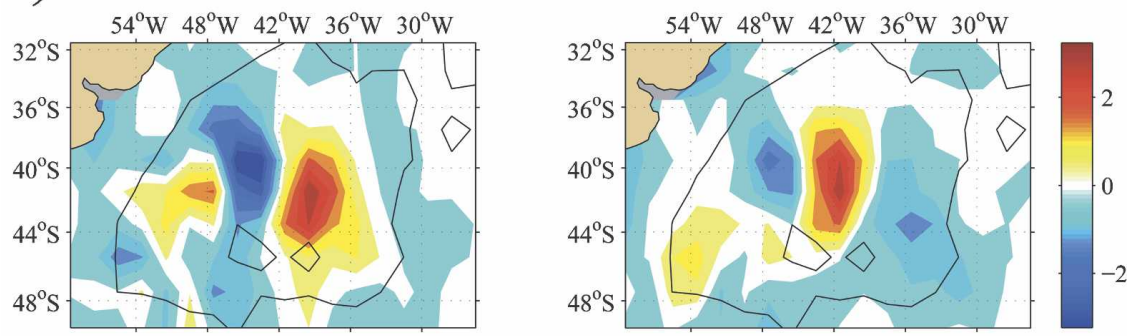

b)
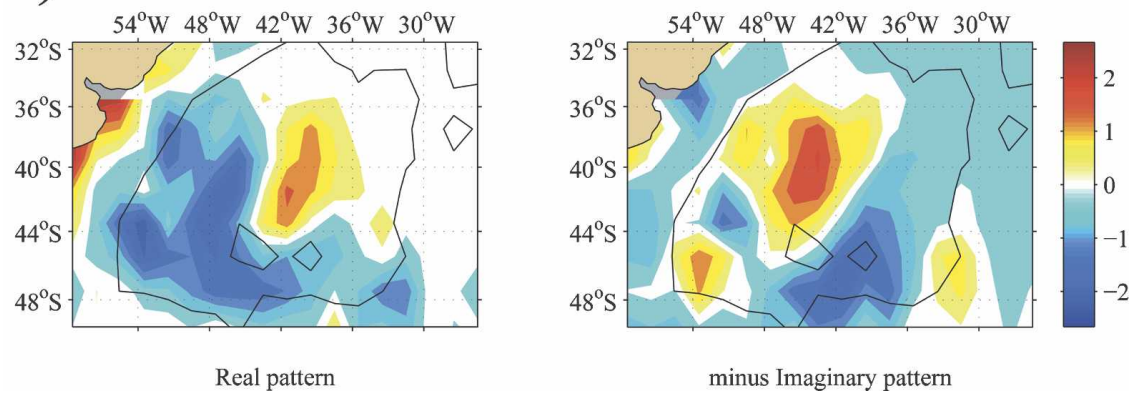

FIG. 9. As in Fig. 3, but now filtered for the period range between 30 and 35 days.

sume that the latter failed to detect an existing 25-day barotropic normal mode. However, there is no obvious reason why a mode that is so similar in structure to modes 1 and 4 could not be found, especially considering its large-scale structure, and the fact that its excitability would reflect a small decay rate comparable to the other modes.

Another possibility is that the existing modes are involved in the response at frequency intervals away from their natural oscillation frequency. The projections of the dynamical modes on SSH data indeed show that the signature of especially modes 1 and 4 are present for a rather broad frequency range (Figs. 5 and 6). In addition, the 25-day statistical mode found by FCQ in altimeter data shares its large-scale bipolar structure with normal modes 1 and 4, while a closer inspection suggests that its position (center of rotation) matches that of mode 4 , rather than mode 1.

In support of this hypothesis, the forced model features several POPs in the 20-25-day range that cannot be readily associated with the free barotropic modes of the normal-mode analysis. Although neither of these statistical modes matches the large-scale bipolar structure or the 25-day period of the statistical mode found by FCQ, the mechanism of generation might be similar. Possible mechanisms that could be responsible are forced excitation of modes by the wind stress, or coupling of modes by bathymetry. Internal dynamics (nonlinear interaction) can be ruled out; repeating the time integration without nonlinear advection terms yielded no noticeable change in the model response.

Another possibility is suggested by the work of Farrell and Ioannou (1993a,b), who show that a nonnormal system, that is, a system whose "normal" modes are not orthogonal, can maintain levels of variance that are higher than can be expected from the presence of damped modes alone. The basic idea of nonnormal growth is that the projection of a small initial perturbation onto a set of nonorthogonal modes can have huge projection coefficients that largely cancel out. When this system evolves, the highly damped modes decay, leaving the projections on the weakly damped modes unbalanced, leading to initial growth of the perturbation, until finally the weakly damped mode also decays (Farrell and Ioannou 1996).

The question is whether these mechanisms are capable of generating a signal that is so persistent in its spatial pattern and oscillation period as the 25-day mode observed by FCQ and Tai and Fu (2005). A mechanism that would account for this persistence is an interaction between normal mode 4 and the Zapiola anticyclone. In Part II this hypothesis is addressed by investigating the sensitivity of mode 4 with respect to an anticyclonic circulation around Zapiola Rise. For this analysis, a Gaussian-shaped anticyclone is prescribed, with $5^{\circ}$ half-width and characteristic velocities of $O\left(10^{-1} \mathrm{~m} \mathrm{~s}^{-1}\right)$ (Saunders and King 1995b). Although this background flow indeed reduces the oscillation pe- 
riod of mode 4 (from 31.5 to 29.7 days), an anticyclone a factor 3.5 stronger would be required to bring this period close to 25 days.

An additional problem with these theories is the fact that a 25-day signal seems to be absent from the bottom-pressure records of Hughes et al. (2007), despite the ideal positioning of these pressure gauges with respect to the dipole structure of the 25-day mode. A final possible explanation is hence that this mode involves physics that is not represented in this barotropic model. The most obvious omission is stratification, and it is conceivable that in essence barotropic modes become surface intensified when stratification is taken into account. It is clear that more work needs to be done to unravel the dynamics of the 25-day mode.

In addition to the rotating modes, both the CEOF and POP analyses identified a northwestward propagating statistical mode north of Zapiola Rise. It is possible that this mode reflects a meandering of the subantarctic (SAF) and subtropical (STF) frontal systems, and captures processes similar to the waves observed by Garzoli and Simionato (1990) at the Brazil-Malvinas Confluence.

The results of this investigation may be relevant to other parts of the ocean as well. Studies using altimeter data (Fu and Smith 1996) and numerical models (Fukumori et al. 1998) have shown that several basins in the Southern Ocean display enhanced levels of variability comparable to the Argentine Basin. These basins, most notably the Bellingshausen Basin (Webb and De Cuevas 2002a) and the Australia-Antarctic Basin (Webb and De Cuevas 2002b), are geographically less well confined. In addition, they appear to house nonoscillatory modes (Webb and De Cuevas 2003): these modes tend to decay exponentially, with no oscillatory component. A detailed study of these basins may reveal why no comparable oscillatory signals seem to exist.

Studying the Argentine Basin has a clear advantage over other basins. The fact that the Argentine abyssal plain is clearly confined by closed contours of $\mathrm{f} / \mathrm{H}$ makes it an ideal laboratory for testing our knowledge of barotropic variability and its representation in numerical models. In addition, the oscillatory character of the variability adds an extra time scale to the problem (oscillation frequency, in addition to decay rate) that can be used to test parameter sensitivities in these models and constrain parameter values. Last, the fact that the oscillation time scale exceeds the Nyquist period of altimeter data (especially the nominal 14-day period of the merged AVISO dataset) allows for a detailed comparison between model results and observations.

\section{Summary and conclusions}

In this paper we addressed the barotropic variability of the Argentine Basin. In particular we considered the oscillatory variability on the time scale of several weeks that has been found in several observational datasets. CEOF and POP analyses on altimeter data and the output of a numerical shallow-water model showed that multiple oscillatory modes may be present in the Argentine Basin. This suggests that conflicting estimates of the period of the variability may be reconciled by the possibility that more than one oscillatory mode can be excited.

A normal-mode analysis of the shallow-water equations confirmed these results by revealing a spectrum of Rossby basin modes of the Argentine Basin. To test whether the eigenmodes really reflect observed variability, we projected the eigenmodes on the data, and performed coherence analysis on the resulting time series. Significant results were found for the first five oscillatory modes, suggesting that they are really excited in the Argentine Basin.

A case was made for the excitation of a rotating mode with a period of 20 days, which is present in most of the observations, and was identified as a barotropic basin mode in this study. However, we could not unambiguously link the 25-day variability as found in altimeter data (Fu et al. 2001) to a free normal mode.

Acknowledgments. This research was supported by NSF through Grant 0424703 (WW, SG, and HD), by NASA JPL Contract 1224031 (SG), and by the Climate Change Prediction Program of the U.S. Department of Energy Office of Science (WW); FV is supported by CNES and CNRS. Los Alamos National Laboratory is operated by the Los Alamos National Security LLC for the National Nuclear Security Administration of the U.S. Department of Energy under Contract DE-AC5206NA25396. This paper benefited greatly from the comments of two anonymous reviewers, and from discussions with Chris Hughes (POL, Liverpool), Glenn Ierley (Scripps, La Jolla), and Georges Weatherly (FSU). The altimeter products were produced by Ssalto/Duacs and distributed by AVISO, with support from CNES.

\section{REFERENCES}

Bretherton, C. S., M. Widmann, W. P. Dymnikov, J. M. Wallace, and I. Bladé, 1999: The effective number of spatial degrees of freedom of a time-varying field. J. Climate, 12, 1990-2009.

De Miranda, A. P., B. Barnier, and W. K. Dewar, 1999: On the dynamics of the Zapiola anticyclone. J. Geophys. Res., 104, 21 137-21 149. 
Dewar, W. K., 1998: Topography and barotropic transport control by bottom friction. J. Mar. Res., 56, 295-328.

Ducet, N., P. Y. L. Traon, and G. Reverdin, 2000: Global highresolution mapping of ocean circulation from TOPEX/ Poseidon and ERS-1 and -2. J. Geophys. Res., 105, $19477-$ 19498.

Farrell, B. F., and P. J. Ioannou, 1993a: Stochastic forcing of the linearized Navier-Stokes equations. Phys. Fluids A, 5, 26002609.

— in unbounded shear and deformation flows. J. Atmos. Sci., 50, 200-211.

— operators. J. Atmos. Sci., 53, 2025-2040.

Fu, L.-L., and R. D. Smith, 1996: Global ocean circulation from satellite altimetry and high-resolution computer simulation. Bull. Amer. Meteor. Soc., 77, 2625-2636.

— B. B. Cheng, and B. Qiu, 2001: 25-day period large-scale oscillations in the Argentine Basin revealed by the TOPEX/ Poseidon altimeter. J. Phys. Oceanogr., 31, 506-517.

Fukumori, I., R. Raghunath, and L.-L. Fu, 1998: Nature of global large-scale sea level variability in relation to atmospheric forcing: A modeling study. J. Geophys. Res., 103, 5493-5512.

Garzoli, S., and C. Simionato, 1990: Baroclinic instabilities and forced oscillations in the Brazil/Malvinas confluence front. Deep-Sea Res., 37, 1053-1074.

Goni, G. J., and I. Wainer, 2001: Investigation of the Brazil Current front variability from altimeter data. J. Geophys. Res., 106, 31 117-31 128.

Hasselmann, K., 1988: PIPs and POPs: The reduction of complex dynamical systems using principal interaction and oscillation patterns. J. Geophys. Res., 93, 11 015-11 021.

Hughes, C. W., V. N. Stepanov, L.-L. Fu, B. Barnier, and G. W. Hargreaves, 2007: Three forms of variability in Argentine Basin ocean bottom pressure. J. Geophys. Res., 112, 1464, doi:10.1029/2006JC003679.

Longuet-Higgins, M. S., 1964: Planetary waves on a rotating sphere. Proc. Roy. Soc. London, A279, 446-473.

Milliff, R. F., J. Morzel, D. B. Chelton, and M. H. Freilich, 2004: Wind stress curl and wind stress divergence biases from rain effects on QSCAT surface wind retrievals. J. Atmos. Oceanic Technol., 21, 1216-1231.

Saunders, P. M., and B. A. King, 1995a: Oceanic fluxes on the WOCE A11 section. J. Phys. Oceanogr., 25, 1942-1958.

, and —, 1995b: Bottom currents derived from shipborne ADCP on WOCE cruise A11 in the South Atlantic. J. Phys. Oceanogr., 25, 329-347.
Schmeits, M. J., and H. A. Dijkstra, 2000: Physics of the 9-month variability in the Gulf Stream region: Combining data and dynamical systems analysis. J. Phys. Oceanogr., 30, 19671987.

Schnur, R., G. Schmitz, N. Grieger, and H. Von Storch, 1993: Normal modes of the atmosphere as estimated by principal oscillation patterns and derived from quasigeostrophic theory. J. Atmos. Sci., 50, 2386-2400.

Sleijpen, G. L. G., and H. A. Van der Vorst, 1996: A JacobiDavidson iteration method for linear eigenvalue problems. SIAM J. Matrix Anal. Appl., 17, 410-425.

Tai, C.-K., and L.-L. Fu, 2005: 25-day period large-scale oscillations in the Argentine Basin revisited. J. Phys. Oceanogr., 35, 1473-1479.

Thomson, J. D., 1982: Spectrum estimation and harmonic analysis. Proc. IEEE, 70, 1055-1096.

Vivier, F., C. Provost, and M. P. Meredith, 2001: Remote and local forcing in the Brazil/Malvinas region. J. Phys. Oceanogr., 31, 892-913.

Von Storch, H., and F. W. Zwiers, 1999: Statistical Analysis in Climate Research. Cambridge University Press, 484 pp.

_, T. Bruns, I. Fischer-Bruns, and K. F. Hasselmann, 1988: Principal oscillation pattern analysis of the 30- to 60-day oscillation in a general circulation model equatorial troposphere. J. Geophys. Res., 93, $11022-11036$.

Weatherly, G. L., 1993: On deep-current and hydrographic observations from a mudwave region and elsewhere in the Argentine Basin. Deep-Sea Res. II, 40, 939-961.

Webb, D. J., and B. A. De Cuevas, 2002a: An ocean resonance in the southeast Pacific. Geophys. Res. Lett., 29, 1252, doi:10.1029/2001GL014259.

- and - 2002b: An ocean resonance in the Indian sector of the Southern Ocean. Geophys. Res. Lett., 29, 1664, doi:10.1029/2002GL015270.

$\longrightarrow$, and $—$ 2003: The region of large sea surface height variability in the southeast Pacific Ocean. J. Phys. Oceanogr., 33, 1044-1056.

Weijer, W., F. Vivier, S. T. Gille, and H. A. Dijkstra, 2007: Multiple oscillatory modes of the Argentine Basin. Part II: The spectral origin of basin modes. J. Phys. Oceanogr., 37, 28692881.

Wunsch, C., and D. Stammer, 1995: The global frequencywavenumber spectrum of oceanic variability estimated from TOPEX/Poseidon altimetric measurements. J. Geophys. Res., 100, 24 895-24 910. 